\title{
Integrating Protein Interaction Networks and Somatic Mutation Data to Detect Driver Modules in Pan-Cancer
}

Hao Wu ( $D$ haowu@sdu.edu.cn )

Shandong University https://orcid.org/0000-0003-2340-9258

\section{Zhong-Li Chen}

Northwest A\&F University: Northwest Agriculture and Forestry University

\section{Ying-Fu Wu}

Northwest A\&F University: Northwest Agriculture and Forestry University

\section{Hong-Ming Zhang}

Northwest A\&F University: Northwest Agriculture and Forestry University

\section{Quan-Zhong Liu}

Northwest A\&F University: Northwest Agriculture and Forestry University

\section{Research}

Keywords: driver modules, node similarity, random walk with restart, complex networks

Posted Date: May 5th, 2021

DOl: https://doi.org/10.21203/rs.3.rs-482282/v1

License: (c) (i) This work is licensed under a Creative Commons Attribution 4.0 International License. Read Full License

Version of Record: A version of this preprint was published at Interdisciplinary Sciences: Computational Life Sciences on September 7th, 2021. See the published version at https://doi.org/10.1007/s12539-02100475-y. 


\title{
Integrating Protein Interaction Networks and Somatic Mutation Data to Detect Driver Modules in Pan-cancer
}

\author{
Hao $\mathrm{Wu}^{2 *}$, Zhong-Li Chen ${ }^{1,3 \dagger}$, Ying-Fu Wu ${ }^{1}$, Hong-Ming Zhang ${ }^{*}$ and Quan-Zhong Liu ${ }^{1}$
}

Sample of title note

\footnotetext{
${ }^{*}$ Correspondence:

haowu@sdu.edu.cn;

zhm@nwsuaf.edu.cn

${ }^{1}$ College of Information

Engineering, Northwest A\&F

University, Yangling, 712100,

China

Full list of author information is

available at the end of the article

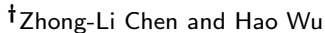

contributed equally to this work.
}

\begin{abstract}
Background: With the constant update of large-scale sequencing data and the continuous improvement of cancer genomics data such as the cancer genome atlas ICGC and TCGA, it gains increasing importance how to detect the functional high-frequency mutation gene set in cells that causes cancer within the field of medicine.
\end{abstract}

Methods: In this study, to solve the issue of mutated gene heterogeneity and improve the accuracy of driver modules, we propose a new recognition method of driver modules, named ECSWalk, based on the human protein interaction networks and pan-cancer somatic mutation data. This study firstly utilizes high mutual exclusivity and high coverage between mutation genes and topological structure similarity of the nodes in complex networks to calculate interaction weights between genes. Secondly, the method of random walk with restart is utilized to construct a weighted directed network, and the strong connectivity principle of the directed graph is utilized to create the initial candidate modules with a certain number of genes. Finally, the large modules in the candidate modules are reasonably split using the way of the induced subgraph, and the small modules are expanded using a greedy strategy to obtain the optimal driver modules.

Results: This method is applied to the analysis of TCGA pan-cancer data, and the experimental results show that ECSWalk can detect driver modules more effectively and accurately, and can identify new candidate gene sets with higher biological relevance and statistical significance than MEXCOWalk and HotNet2.

Conclusions: ECSWalk is of theoretical guidance and practical value for cancer diagnosis, treatment and drug targets.

Keywords: driver modules; node similarity; random walk with restart; complex networks

\section{Introduction}

2 In recent years, with the continuous advancement of cancer research at the bio- 
4 area in cancer [1]. For the driver genes that have functional mutations in cancer, 5 specific drugs can be used to control their expression and transcription levels, and 6 effectively control the development and deterioration of cancer. However, we cannot 7 effectively identify all driver mutations in cancer by statistical analysis of a single $s$ mutation gene[2]. Therefore, compared with the screening of a single driver muta9 tion gene, detection of the mutated driver gene sets in cancer is of high biological relevance and statistical significance [3, 4]. In particular, they can not only explore 11 the pathogenesis of cancer in depth, but also provide drug targets for the clinical 12 treatment of cancer by inferring the interaction of upstream and downstream genes 13 in the driver modules, and they provide reliable theoretical basis and data support 14 for precision medicine and personalized medicine [5]. Previous studies have found that screening for high-frequency mutations is con16 ducive to identifying the group of mutated driver genes in cancer $[6,7,8]$, such 17 as EGFR, TP53, PIK3CA and other high-frequency mutation genes, which are screened as driver factors during tumorigenesis. However, it normally involves a large amount of time and effort to identify high-frequency mutation gene sites in

a large number of tumor samples for biological experimental verification, which is

difficult to achieve under the current technical level, experimental conditions, and

research capabilities. Also, the current methods tend to ignore the problem of mutation heterogeneity in complex diseases $[9,10]$. To effectively solve this problem, most of the previous studies utilized the high mutual exclusivity and high coverage widely existed in the genome map to explore the driver pathways that lead to the occurrence of cancer $[6,7,11,12]$. 
Based on the characteristics of high mutual exclusivity and high coverage, Vandin et al.[6] proposed the Dendrix algorithm to identify driver pathways from somatic mutation data. This method introduces a penalty overlap and a reward coverage mechanism to solve the maximum weight sub-matrix problem of driver pathway identification based on gene mutation data. The Dendrix algorithm not only improves coverage but also guarantees mutual exclusivity among the genes in a driver pathway. However, this iterative search method is prone to produce local optimal solutions, and it requires specifying the number of genes in a driver pathway in advance. Leiserson et al.[7]proposed a Multi-Dendrix algorithm based on the Dendrix algorithm, which detects multiple driver pathways at the same time. The algorithm utilizes linear regression to simultaneously detect multiple gene sets that meet high coverage and high mutual exclusivity, and can generate a globally optimal solution. However, the Multi-Dendrix algorithm needs to pre-specify both the maximum number of genes in a driver pathway and the number of driver pathways.

Therefore, the two algorithms do not have good universality and robustness, and have certain limitations when applied to different data sets.

Pan-cancer data analysis provides new ideas and methods for the study of clinical diagnosis and treatment across cancer types $[13,14,15,16]$. As one application, Leiserson et al.[14] proposed the HotNet2 algorithm by integrating differential expression genes, significant mutation genes and protein interaction networks to detect combinations of rare somatic mutations. Using the principle of thermal diffusion and the random walk with restart model, the edge weight that becomes stable after the random walk is restarted as the weight of the directed edge. By removing the di- 
rected edge with a small weight, the method detects strongly connected components as driver modules in the directed graph. Although the algorithm reduces the output of false positive results and improves the accuracy of the prediction results, the conversion probability just considers the degree of the vertex during the random walk process, but ignores the mutual exclusivity among genes. Therefore, the problem of gene mutation heterogeneity cannot be effectively solved in the pan-cancer data with large differences.

Based on the HotNet2 algorithm, Rafsan et al. [16]proposed a MEXCOWalk algorithm to mine driver modules by using split and expansion techniques. The algorithm utilizes mutual exclusivity between genes and coverage scores of gene set to reflect the edge weight of the network. Then the random walk with restart strategy is used to construct a weighted directed network, and the split and expansion techniques are utilized to mine driver modules. Although the set of driver genes identified by this algorithm has high mutual exclusivity and high coverage, the algorithm ignores the relationships of mutual exclusivity between expanded leaf nodes and seed modules in the expansion stage of small modules. Therefore, this algorithm reduces the accuracy of driver module identification to a certain extent.

As mentioned above, although the previous methods can detect the gene sets with high mutual exclusivity and high coverage, they just focus on the mutual exclusivity and coverage between genes, instead of the topological structure of complex networks. To effectively solve the problem of mutated gene heterogeneity and improve the accuracy of driver modules, this paper proposes a driver module detection algorithm (ECSWalk) based on gene mutation and human protein interaction network. 
The algorithm optimizes the definition in terms of the following three characteris-

tics of high mutual exclusivity, high coverage among genes and high similarity of topological structure. Firstly, the complex network topology analysis method is used in the human protein interaction network data to calculate the topology similarity between network nodes, and then it is combined with the two characteristics of high coverage and high mutual exclusivity of the mutated genes to obtain the weight of the vertices and edges in the human protein network. Among them, the weights of vertices in the human protein network are obtained according to the coverage of mutated gene; the random walk with restart strategy is utilized to calculate the weights of edges in the network by the three characteristics, namely the coverage, the mutual exclusivity, and the similarity of the topological structure between the nodes. Secondly, based on the weighted network constructed in the previous step, the large modules are split into several candidate gene sets using the method of the induced subgraph. And the greedy strategy is utilized to add the nodes in the leaf module to the seed module to achieve the optimal gene sets. These mutated gene sets with high mutual exclusivity, high coverage and high similarity of the topological structure are likely to work as driver modules in cancer[17, 18]. This study not only applies the analysis method of complex network topology to the biological network, but also improves the method of determining module size based on split and expansion. The study clarifies the interactions between genes in the detected driver modules, which promote the study of cancer pathogenesis and drug targets. 
9

95

96

97

98

\section{Methods}

Mutual exclusivity and coverage

To accurately detect and classify a large number of genes in the cancer genome map, and reduce the impact from error in the actual biological sequencing experiment process, this study introduces the definition of mutual exclusivity and coverage[16].

Let $G(V, E)$ denote the protein interaction network (PPI), each vertex $u_{i} \in V$ corresponds to a protein in PPI network, and each protein $u_{i}$ corresponds to a mutated gene $g_{i}$. The undirected edge $\left(u_{i}, u_{j}\right) \in E$ in PPI network corresponds to the interaction between gene pair $\left(g_{i}, g_{j}\right)$. Therefore, the node $g_{i}$ represents both a gene and the corresponding protein in $G$. The sample with gene $g_{i}$ mutated is represented by $S_{i}$, and $M \subseteq V$ is a subset of genes. For any pair of genes $g_{i}, g_{j} \in M$, $g_{i} \neq g_{j}$, if $S_{i} \cap S_{j}=\varnothing$, the genes in $M$ are mutually exclusive.

The mutual exclusivity of gene subset $M$ is represented as:

$$
E D(M)=\frac{\left|\cup_{\forall g_{i} \in M} S_{i}\right|}{\sum_{\forall g_{i} \in M}\left|S_{i}\right|}
$$

106 if $E D(M)=1$, then the genes in the subset $M$ are mutually exclusive, that is, at most one gene within the subset $M$ is mutated in each sample.

The coverage of gene subset $M$ is represented as:

$$
C D(M)=\frac{\left|\cup_{\forall g_{i} \in M} S_{i}\right|}{\left|\cup_{\forall g_{i} \in V} S_{i}\right|}
$$


If $C D(M)=1$, then the gene subset $M$ completely covers all patients, that is, at least one gene within the subset $M$ is mutated in each sample.

Node similarity

The abnormal local area network composed of nodes and their neighbor nodes may affect the performance of the entire network in complex networks. Therefore, to measure the topological relationship of each mutated gene in the same driver module, we select the local area network with node $g_{i}$ as the center and its direct neighbor node as the radius. Combined with the characteristics of the local area network structure, we propose node similarity based on Jensen-Shannon (JS) divergence and analyze the topological structure of the protein interaction network $[19,20,21]$. The similarity is mainly defined by the discrete probability set, and the index construction process mainly includes two steps, the first step is to construct the probability set[22], and the second step is to define the node similarity according to the JS divergence.

Construction of the probability set. Let $d_{i}$ be the degree of the $i$ th gene node, and $d_{\max }$ be the maximum node degree in the local area network. Suppose there are $N$ nodes in the probability set of one node, $N=d_{\max }+1$. The sum of node degrees $D_{g_{i}}$ of gene node $g_{i}$ in its local area network is expressed as follows:

$$
D_{g_{i}}=\sum_{j=1}^{n} d(j)
$$
of the $j$ th gene in the local area network of gene $g_{i}$. 
In the local area network, the discrete probability of gene $g_{i}$ is expressed as follows:

$$
p(i)=\frac{d_{i}}{D_{g_{i}}}
$$

$$
P(i)=\left(p_{i}(1), p_{i}(2), \ldots, p_{i}(n), \ldots, p_{i}(N)\right)
$$

Suppose that two adjacent genes $g_{i}$ and $g_{j}$ in the constructed gene network correspond to two different probability sets $P(i)$ and $P(j)$ with the same number of genes in the local area network. According to the set of discrete probability constructed in formula (5), the Kullback-Leibler (KL) divergence value between genes $g_{i}$ and $g_{j}$ is expressed as:

$$
D_{K L}(P(i) \| P(j))=\sum_{k=1}^{N} p_{i}(k) \log \frac{p_{i}(k)}{p_{j}(k)}
$$


Due to the asymmetry of KL divergence, different results can be obtained by exchanging the positions of $P(i)$ and $P(j)$. To solve this asymmetry problem, the same result can be obtained by exchanging the positions of $P(i)$ and $P(j)$, The JS divergence value is obtained based on the KL divergence value, which is expressed as:

$$
D_{J S}(P(i) \| P(j))=\frac{1}{2} K L\left(P(i) \| \frac{P(i)+P(j)}{2}\right)+\frac{1}{2} K L\left(P(j) \| \frac{P(i)+P(j)}{2}\right)
$$

Therefore, we use an analysis of gene pair similarity based on the network topology, which is defined as follows:

$$
\operatorname{SIM}\left(g_{i}, g_{j}\right)=1-D_{J S}(P(i) \| P(j))
$$

\section{Construction of edge-weighted networks}

Given a PPI network $G(V, E)$, where node set $V=\left(u_{1}, u_{2}, u_{3}, \ldots, u_{n}\right)$ represents the set of mutated genes corresponding to the PPI network, edge set $E=\left\{e=\left(u_{i}, u_{j}\right)\right\}$ represents the set of protein interaction relationships.

Construction of a weighted undirected graph $G_{\omega}$. For each vertex $g_{i} \in V$, the coverage of vertex $g_{i}$ represents the weight of vertex $g_{i}$, that is, $\omega\left(g_{i}\right)=C D\left(g_{i}\right)$. 
Obviously, the more the mutated samples in gene $g_{i}$, the greater the weight of the vertex $g_{i}$.

Taking into account the chance of increasing the coexistence of a gene and its surrounding genes, this study defines the set of node $g_{i}$ and its direct neighbor nodes as the local area network $N e\left(g_{i}\right)$ as follows:

$$
N e\left(g_{i}\right)=\left\{g_{i}\right\} \bigcup\left(\cup_{\forall\left(g_{i}, g_{j}\right) \in E} g_{j}\right)
$$

To balance the mutual exclusivity between genes and the opportunities for the coexistence between a gene and its surrounding genes, this study utilizes the average value of $E D\left(N e\left(g_{i}\right)\right)$ and $E D\left(N e\left(g_{j}\right)\right)$ as the mutual exclusivity $E D\left(g_{i}, g_{j}\right)$ of gene pairs in the network, as shown below.

$$
E D\left(g_{i}, g_{j}\right)=\frac{E D\left(N e\left(g_{i}\right)\right)+E D\left(N e\left(g_{j}\right)\right)}{2}
$$

To reduce the chance of a single gene with large coverage added to the edge weight, the product of the coverage of two genes is used to represent the coverage $C D\left(g_{i}, g_{j}\right)$ between gene pairs, as shown below.

$$
C D\left(g_{i}, g_{j}\right)=C D\left(\left\{g_{i}\right\}\right) \times C D\left(\left\{g_{j}\right\}\right)
$$

The study integrates the three characteristics of mutual exclusivity, coverage, and similarity among gene pairs to calculate the edge weight of the weighted undirected 
graph as follows:

$$
\omega\left(g_{i}, g_{j}\right)=\left\{\begin{array}{cc} 
& \operatorname{SIM}\left(g_{i}, g_{j}\right) \neq 0 \\
\frac{2 \times S I M\left(g_{i}, g_{j}\right)}{\frac{1}{E D\left(g_{i}, g_{j}\right)}+\frac{1}{C D\left(g_{i}, g_{j}\right)}} & E D\left(g_{i}, g_{j}\right) \neq 0 \\
& C D\left(g_{i}, g_{j}\right) \neq 0 \\
0 & \text { otherwise }
\end{array}\right.
$$

The principle of thermal diffusion is utilized to construct a weighted directed graph by performing a random walk with restart on $G_{\omega}[14]$. The random walk with restart means that the source node gene $g_{i}$ transfers to its neighboring nodes with a certain probability, and they utilize the restart probability to transfer to the source node again. This process is repeated until it reaches a stable state. It is expressed as follows:

$$
F_{t+1}=(1-\beta) P F_{t}+\beta F_{0}
$$

Where $F_{0}$ is the initial state of the source node gene $g_{i}$, and $F_{0}=C D\left(g_{i}\right) \cdot F_{t}$ is the probability distribution at time $t ; \beta$ is the restart probability of its neighbor nodes transferring to the source node, and $0 \leqslant \beta \leqslant 1$, which is used to control the heat of the source node diffusion to the rest nodes of the network. It is necessary to choose a suitable $\beta$ that all source nodes retain most of the heat in their direct neighbor nodes [14]. According to $[14,16]$, the value of $\beta$ in the study is set to be $0.4 ; E$ represents the transition probability matrix of the restart random walk 
process, which is positively correlated with the edge weight, as shown below:

$$
P\left(g_{i}, g_{j}\right)=\left\{\begin{array}{cc}
\frac{\omega\left(g_{i}, g_{j}\right)}{\sum_{k} \omega\left(g_{i}, g_{j}\right)} & \left(g_{i}, g_{j}\right) \in E \\
0 & \text { otherwise }
\end{array}\right.
$$
follows.
Where $I$ is the identity matrix. Restart the random walk to create a directed edge with weight $F$ for each pair of gene pair $g_{i}$ and $g_{j}(i \neq j)[14]$, and finally realize the construction of a weighted directed graph $G_{d}$. The algorithm is described as ows.

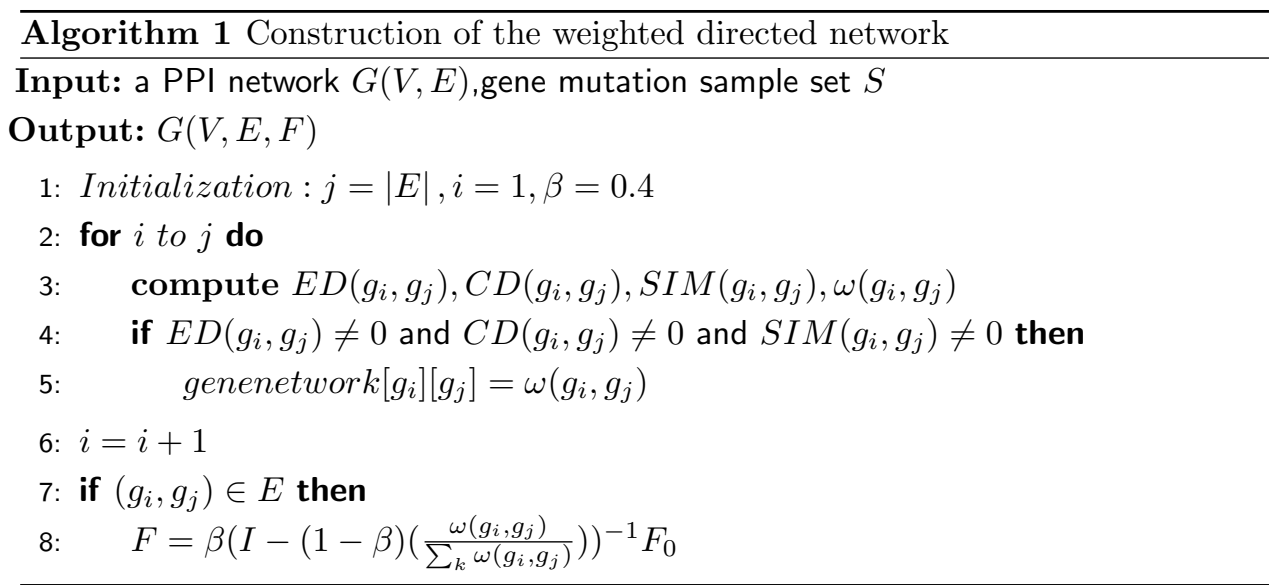




\section{Driver modules detection}

In this study, the strongly connected component (SCC) division method of the directed graph is utilized to generate the driver modules [14]. The process is divided into three steps.

The first step is to create a set of initial candidate modules. The SCC is firstly employed as an initial set of candidate modules. The minimum weight edge in $G_{d}$ is iteratively deleted until strongly connected subgraphs are generated from $G_{d}$, and then the strongly connected subgraph is added to the initial module set $P$. Finally, all the modules in $P$ whose gene number is less than min_module_size are removed.

The above process is carried out iteratively until the number of genes in $P$ decreases to total_genes. We finally obtain the initial module set $P=\left(M_{1}, M_{2}, \ldots, M_{r}\right)$.

The second step is to split the large and medium-sized modules into module set $P$ [16]. For the weighted directed graph $G_{d}$ and a module $M_{q}, G_{d}\left(M_{q}\right)$ denotes the gene set of derived subgraphs in the directed graph $G_{d}$ (the derived subgraph is different from the strongly connected subgraph), which corresponds to the genes in $M_{q} . L$ denotes the set of derived subgraphs, as shown below.

$$
L=\left\{G_{d}\left(M_{q}\right)\right\}
$$

Let split_size be the degree of the node with the largest value in the subgraph derived by module $M_{q}$, Modules with more nodes than split_size will be split as large modules. In the splitting process, $G_{c} \in L$ is a subgraph derived from directed graph $M_{q}, v^{\prime}$ is the node with the largest out-degree value in $G_{c}$, and $I N\left(v^{\prime}\right)$ 
represents the local area network of $v^{\prime}$ in $G_{c}$. If the number of nodes in $I N\left(v^{\prime}\right)$ is not less than min_module_size, then they will be divided into seed modules, or else they will be divided into leaf modules. All the strongly connected subgraphs that meet the conditions in the directed graph $G_{c}$ are split in the same way.

The third step is to add leaf modules to the seed module. The leaf node $g_{m}$ connected to any node in the seed module is selected to extend the seed module by utilizing the greedy strategy, and the extension function is defined as follows:

$$
G\left(g_{m}\right)=\overline{G^{\text {in }}\left(g_{m}\right)}-\overline{G^{\text {out }}\left(g_{m}\right)}
$$

\footnotetext{
Where $\overline{G^{i n}\left(g_{m}\right)}$ represents the average weight of the edge between node $g_{m}$ in the leaf module and the node in the seed module, $\overline{G^{\text {out }}\left(g_{m}\right)}$ represents the average weight of the edge between node $g_{m}$ in the leaf module and the rest of the nodes in the leaf module. If $\overline{G^{\text {in }}\left(g_{m}\right)}$ is not less than $\overline{G^{\text {out }}\left(g_{m}\right)}$, then the node is added to the seed module.
}

\section{Experimental results and analysis}

\section{Data preprocessing}

This study utilizes the somatic mutation data and the combined human PPI network data from HINT+HI2012 [14]. Somatic mutation data comes from the TCGA pan-cancer dataset containing 12 cancer types, which are composed of 3281 samples with 20472 SNVs and 4334 samples with 720 CNAs. According to the data preprocessing method of [14], the samples with hyper-mutation and the genes that are low-expression in all tumor types are firstly screened out. And then MutSigCV 


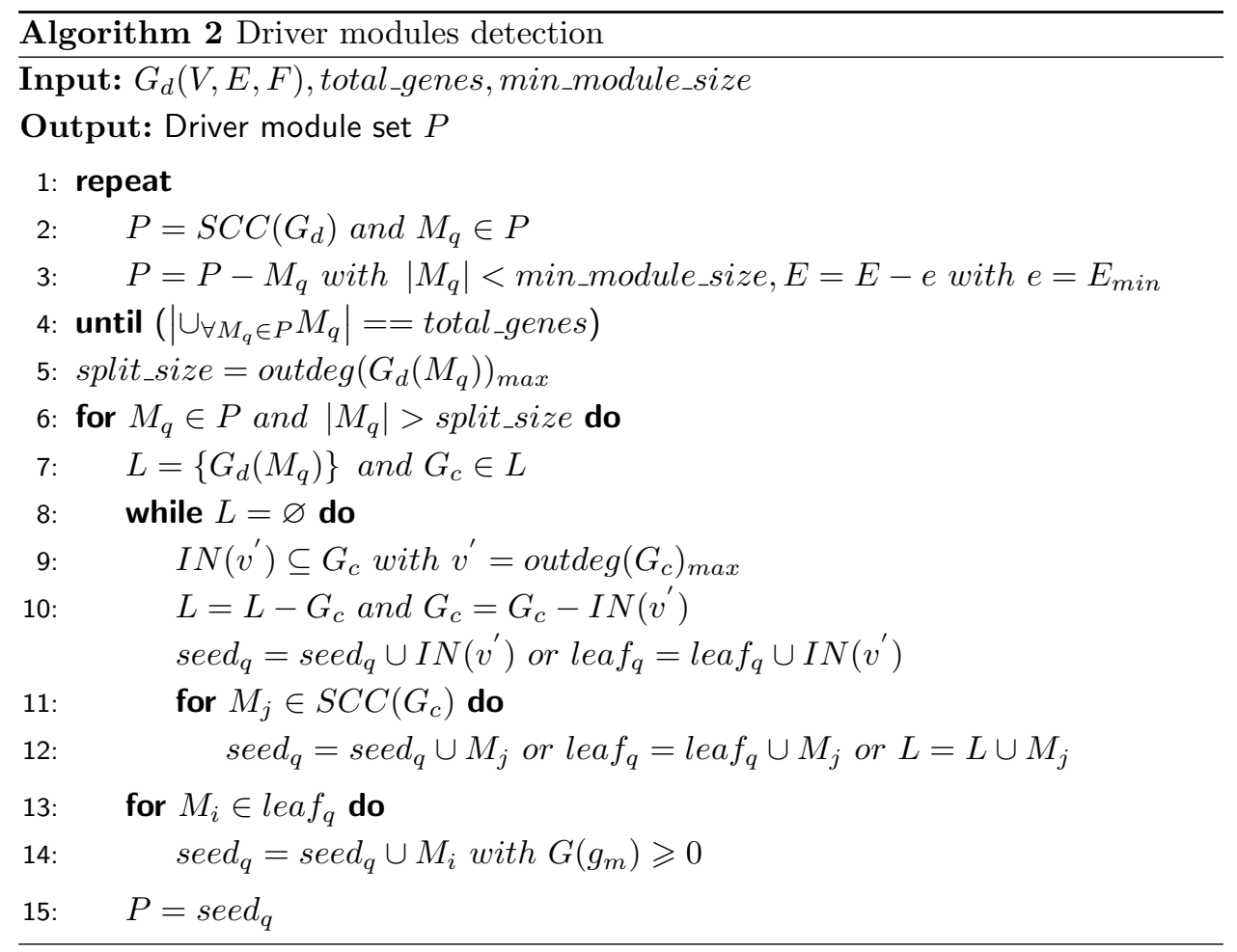

tool is applied to filter the genes without obvious mutations in SNVs, after that 218

genes are deleted, 5 samples without SNV and 1973 samples with only CNA are deleted, and 7894 genes with $<3$ RNA-seq reads in $>30 \%$ of tumors of each cancer type are deleted. Finally, we obtain the data set containing 3110 samples and a total of 11565 somatic mutation genes. (2) HINT+HI2012 as the PPI network data includes high-quality interaction database (HINT) and human interaction database (HI2012). The data preprocessing is as follows, a merge operation is firstly performed based on the interaction relationship in the HINT and HI2012 database. Then, we delete closed loops and duplicate edges in the new PPI network. Finally, we obtain a protein interaction network composed of 9858 proteins and 40704 interactions. 


\section{Parameter setting}

If the number of genes in a driver module is less than 3 , the gene set is not usually considered as a driver module $[10,14]$. Therefore, min_module_size as the minimum module size is set to be 3 .

\section{Enrichment analysis}

To verify the enrichment effect of the modules identified by ECSWalk, we utilize functional annotation tools (DAVID) to analyze the enrichment of the driver modules detected by ECSWalk, HotNet2, and MEXCOwalk algorithm. The results in nine types of cancer are shown in Figure 1. The value is set to be 100 to obtain the driver modules in the ECSWalk and MEXCOWalk, and HotNet2 obtains 14 consensus driver modules.

As shown in Figure 1, the enrichment effect of the driver modules detected by ECSWalk is better than that by HotNet2 and MEXCOWalk algorithms among the nine types of cancer, especially in GBM, Melanoma, UCEC, NSCLC, PAAD and CML. Therefore, the driver modules identified by ECSWalk show extremely high statistical significance and biological relevance.

\section{Comparison of module accuracy}

To evaluate the accuracy of the driver modules detected by ECSWalk, this study uses the Accuracy and F-measure evaluation indices to measure the accuracy of the driver modules based on the known pathways[24]. The higher the Accuracy value, the better the classification effect. The higher the F-measure value, the more driver modules can be enriched in the known biological pathways, indicating that 
the method is more accurate in mining driver modules. The calculation formulas of Accuracy and F-measure are as follows:

$$
\text { Precision }=\frac{T P}{T P+F P}
$$

$$
\text { Recall }=\frac{T P}{T P+F N}
$$

$$
F-\text { measure }=2 \times \frac{\text { Precision } \times \text { Recall }}{\text { Precision }+ \text { Recall }}
$$

$$
\text { Accuracy }=\frac{T P+T N}{T P+F P+T N+F N}
$$

Where TP indicates the number of modules in which positive classes are predicted to be positive classes; FP indicates the number of modules in which negative classes are predicted to be positive classes; $T N$ indicates the number of modules in which negative classes are predicted to be negative classes; $F N$ indicates the number of modules in which positive classes are predicted to be negative classes. 
The following formula is utilized to calculate the enrichment of the driver modules in one known biological pathway.

$$
p-\text { value }=\frac{\left(\begin{array}{c}
K \\
k
\end{array}\right)\left(\begin{array}{c}
N-K \\
n-k
\end{array}\right)}{\left(\begin{array}{l}
N \\
n
\end{array}\right)}
$$

\footnotetext{
Where $N$ represents the total number of genes, $K$ represents the number of genes in a known biological pathway, $n$ represents the number of genes in a driver module, and $k$ represents the number of overlapping genes between a known biological pathway overlaps and a driver module. The driver modules with p-value $<0.01$ are set to be the positive type, and the driver modules with $\mathrm{p}$-value $\geqslant 0.01$ are set to be the negative type. All the p-values are corrected using the Benjamin-Hochberg method.
}

Figure 2 shows the enrichment performance of the driver modules obtained by ECSWalk, MEXCOWalk and HotNet2 based on the known biological pathways obtained by using the DAVID tool for enrichment analysis. It can be seen from Figure 2A, the ACC value of the ECSWalk is 1 in the nine types of cancer, which shows that ECSWalk has extremely high accuracy in detecting driver modules. Specifically, the ACC value of the ECSWalk is $100 \%, 200 \%$ and $50 \%$ higher than that of the HotNet2 respectively in the three cancers of GBM, BLCA and PAAD, and the ACC value of the ECSWalk is $50 \%, 200 \%, 50 \%, 33.3 \%, 100 \%$ and $100 \%$ higher than MEXCOWalk respectively in GBM, BLCA, UCEC, NSCLC, PAAD and LAML. Also of note, the ACC value of the ECSWalk is 1, but the ACC values 
of the MEXCOWalk and HotNet2 are 0 in CDAD. Therefore, ECSWalk has better performance than MEXCOWalk and HotNet2 in detecting driver modules in cancer.

As can be seen in Figure 2B, the F-measure values of the ECSWalk algorithm are 1 in the nine types of cancer, which means that the accuracy and recall of the ECSWalk algorithm are both 1 in these nine cancers. This shows all the predicted driver modules are positive. It is interesting to note that the F-measure values of gene sets detected by MEXCOWalk and HotNet2 algorithms are both 0 in CDAD. Therefore, ECSWalk has a better capability in detecting driver modules in nine types of cancers.

\section{Comparison of the optimal modules}

\section{EGFR module}

The weighted diagram of the EGFR module detected by ECSWalk is shown in Figure 3A. The EGFR module detected by ECSWalk, MEXCOWalk and HotNet2 is shown in Table 1.

It can be seen from Table 1, the p-value of the EGFR module detected by ECSWalk is 1.09E-13, and the p-values of the EGFR module detected by MEXCOWalk and HotNet2 are 8.1E-07 and 6.9E-06, respectively. Although the number of genes in the EGFR module detected by ECSWalk is less than that by MEXCOWalk, the EGFR module detected by ECSWalk has higher coverage than those of the other two algorithms. Therefore, the gene set detected by ECSWalk has higher biological relevance and statistical significance than those by the other two algorithms.

The EGFR module detected by ECSWalk mutates in $62.77 \%(1952 / 3110)$ samples, in which PIK3CA, EGFR, APC, ERBB2 and PIK3R1 have high mutation 
frequency, and mutate in $19.36 \%, 8.39 \%, 7.52 \%, 6.37 \%$ and $4.98 \%$ samples respec-

tively. This module is enriched in a variety of cancers, especially the p-value of this module in UCEC is $1.09 \mathrm{E}-13$ according to the DAVID enrichment analysis. It can be also seen from Figure 3 that PIK3CA and NRAS, PIK3R1 have large edge weights, and PIK3R1 and HRAS have a large edge weight, which indicates that the four mutated genes have strong interaction relationships. In the ErbB signaling pathway (Figure 4a), EGFR and IGF1R promote the expression of PIK3CA and PIK3R1, and phosphorylation promotes the expression of HRAS and NRAS. At the same time, HRAS and NRAS promote the expression of PIK3CA and PIK3R1. Besides, CTNNB1 and APC have large weight values, which indicates that the two mutated genes have a strong interaction relationship. In the Wnt signaling pathway (Figure 4b), CTNNB1 promotes the expression of itself and APC. This shows that the mutations of CTNNB1 and APC constitute two key genes in the Wnt/ $\beta$-Catenin pathway, and thus can be used as predictors of cervical cancer susceptibility [25].

\section{PPFIA1 module}

The weighted diagram of the PPFIA1 module detected by ECSWalk is shown in Figure 3B. The PPFIA1 module detected by ECSWalk, MEXCOWalk and HotNet2 is shown in Table 2. HotNet2 does not mine any genes contained in the module, and the gene set detected by ECSWalk contains one more gene PPFIA1 than that by MEXCOwalk. The coverage of this module is $7.72 \%$, and the mutual exclusivity is $96.77 \%$. Although the PPFIA1 modules detected by the three algorithms do not show enrichment information in the DAVID enrichment analysis, studies have shown that there is an interaction between genes within the module [26, 27]. The 
accuracy of the module can be verified in the previous research [27] in which the surface tyrosine phosphatase receptor PTPRF and its adaptor PPFIA1 drive active $\alpha 5 \beta 1$ integrin recycling and controls fibronectin fibrillogenesis and vascular morphogenesis. Besides, the proteins encoded by PTPRF and PTPRS are important members of the PTP family of protein tyrosine phosphatases. PTPS is a signal molecule that regulates cell growth cycle, differentiation process, mitosis, gene mutations and other cell life processes. The lack of PTPRS and PTPRF affects the proliferation of mandibular cells, and results in craniofacial deformities. The WNT and BMP signaling pathways are dysregulated in cells deficient in PTPRS and PTPRF [26]. Therefore, PPFIA1, PTPRS and PTPRF may be mutated in the same pathway and cause cancer. In summary, the PPFIA1 module detected by ECSWalk is of more biological relevance than those by the other two algorithms.

\section{TP53 module}

The weighted diagram of the TP53 module detected by ECSWalk is shown in Figure 3C. The TP53 module detected by ECSWalk, MEXCOWalk and HotNet2 is shown in Table 3. The TP53 module detected by ECSWalk has higher coverage than those by MEXCOWalk and HotNet2. The p-value of the TP53 module detected by ECSWalk is 3.25E-20 according to the DAVID enrichment analysis, and the p-values detected by MEXCOWalk and HotNet2 are 5.6E-08 and 3.84E-06 respectively. Therefore, the gene set detected by ECSWalk has higher biological relevance and statistical significance than those by the other two algorithms.

The TP53 module detected by ECSWalk mutates in $70.09 \%$ (2180/3110) samples. This module has significant enrichment in CML cancer, and the p-value of this mod- 
ule in CML cancer is 1.1E-11 according to the DAVID enrichment analysis, which indicates that the module has high biological relevance. It can be seen from Figure 3C, TP53, ATM, CHEK2, MDM2, MDM4, PTEN, CDKN2A, CDK4 and CDK6 have large edge weights, which indicates that the nine mutated genes have strong relationships. In the P53 signaling pathway (Figure 4d), phosphorylation of ATM promotes the expression of CHEK2, and phosphorylation of CHEK2 promotes the expression of TP53; TP53 inhibits the expression of CDK4 and CDK6 by promoting the expression of CDKN1A, and TP53 promotes the expression of upstream MDM2 and the expression of downstream MDM2 and PTEN; MDM4 promotes the expression of MDM2, but inhibits the expression of TP53; CDKN2A inhibits the expression of MDM2, and MDM2 inhibits the expression of MDM4. This indicates that regulating the p53 signaling pathway can interfere with CML, thereby regulating the occurrence and development of CML [28].

\section{Analysis of the remaining modules}

\section{BAP1 module}

The weighted diagram of the BAP1 module detected by ECSWalk is shown in Figure $3 \mathrm{D}$, and the mutual exclusivity of the BAP1 module is $96.95 \%$. The BAP1 module is a PR-DUB protein complex composed of BAP1 and ASXL1, which activates its downstream tumor suppressor genes such as SOCS1/2, VHL and TXNIP by combining with transcription factors[29]; In blood tumor cells, the BAP1 mutation makes the C-terminal truncated ASXL1 mutation protein completely lose its ability to bind to transcription factors, causes the ASXL1 mutation protein to significantly weaken the transcription and regulation functions of the BAP1-ASXL1-FOXK1/K2 
complex through a dominant negative mutation effect, reduces the expression of tu-

mor suppressor genes, thereby regulating glucose metabolism, hypoxia perception and JAK-STAT and other tumor-related signaling pathways. This thus contributes to promoting the proliferation and self-renewal of leukemia cells, and further inhibiting cell apoptosis under hypoxia [29]. It can be seen that ASXL1 and BAP1 have high biological relevance, and may exist in the same driver pathway to work together and promote the occurrence of cancer.

\section{NOTCH3 module}

The weighted diagram of the NOTCH3 module is shown in Figure 3E. The NOTCH3 gene has high coverage and is altered in 129 samples. The mutual exclusivity of this module is $98.82 \%$, and it has been reported that the NOTCH signaling pathway plays a vital role in the tumor microenvironment, and the ligand genes DLL1 and JAG1 of the NOTCH signaling pathway have mutations in a variety of cancers, such as DLL1, JAG1 and NOTCH3 existing high probability of mutations in patients with colon cancer [30]. Besides, DLL1 and JAG1 can control the rate of neural development in the NOTCH3 gene expression domain, and JAG1 can activate the NOTCH signal transduction in the V1 and DL6 domains. DLL1 can also send signals to nerve cells outside the NOTCH gene expression domain [31]. Therefore, DLL1, JAG1 and NOTCH3 genes may exist in the same driver pathway.

\section{PAF1 module}

The weighted diagram of the PAF1 module detected by ECSWalk is shown in Figure 3F. The PAF1 module detected by ECSWalk mutates in $4.34 \%(135 / 3110)$ sam- 
ples, and the PAF1 gene mutates in 104 samples. The mutual exclusivity between genes in the module is $100 \%$. This module has a significant enrichment relationship in Cdc73/Paf1 complex, and the p-value of this module in Cdc73/Paf1 complex is 2.9E-9 according to GO enrichment analysis, which indicates that the genes in this module have high biological relevance. Besides, CTR9, as the main component of the RTF1 complex, participates in the assembly of the PAF1 complex by the TRP domain, and Cdc73/Paf1 is a polyprotein complex associated with RNA polymerase II and general RNA polymerase II transcription factor complexes[32], and it may be involved in transcription initiation and extension. Furthermore, the mutated genes PAF1 and CTR9 in the Cdc73/Paf1 complex can cause a variety of diseases including malignant tumors. The PAF1 module also has a significant enrichment relationship in Transcription elongation from RNA polymeraseII promoter pathways. The p-value of this module is $2.7 \mathrm{E}-8$ according to GO enrichment analysis. It has been reported in [33] that CTR9, RTF1 and LEO1 are the main members of the Paf1/RNA polymerase II complex, PAF1, Rtf1 and LEO1 have frequent interactions with PAF1, Cdc73 and PolII, and the deletion of PAF1 or CTR9 will lead to similar severe pleiotropic phenotypes. Therefore, the genes in this module have high biological relevance and may exist in the same driver pathway to cause cancer.

\section{MAP3K1 module}

The weighted diagram of the MAP3K1 module detected by ECSWalk is shown in Figure 3G. This module has significant enrichment in the MAPK signaling pathway, and its p-value is 1.3E-3 according to the KEGG enrichment analysis. The phosphorylation of MAP3K1 in this module activates MAP2K4, MAP2K1 and MAP2K2, 
and the phosphorylation of BRAF activates MAP2K1 and MAP2K2. It has been reported in[34] that inhibiting the MAPK pathway can lead to lung cancer cell apoptosis. Therefore, the genes in this module have high biological relevance and may exist in the same driver pathway to cause cancer.

\section{MCL1 module}

The weighted diagram of the MCL1 module detected by ECSWalk is shown in Figure 3H. It has been reported in [35] that USP9X and MCL1 in this module have high biological relevance. For example, USP9X stabilizes the expression of MCL1 and promotes cell survival, and high expression of USP9X leads to an increase in the amount of MCL1 protein in human follicular lymphoma and diffuse large B-cell lymphoma, and vice versa. Therefore, USP9X is usually used as a target for clinical treatment by maintaining the stability of the amount of MCL1 and other proteins in human malignant tumors [35]. It can be seen that MCL1 and USP9X have high biological relevance and may exist in the same driver pathway.

\section{MYC module}

The weighted diagram of the MYC module detected by ECSWalk is shown in Figure 3I. The module has more significant enrichment in the nucleoplasm, and the p-value of this module in nucleoplasm is $8.3 \mathrm{E}-5$ according to GO enrichment analysis, which indicates that this module has high biological relevance. It can be seen from Figure 3I that MYC has the largest coverage in the module, and MYC is altered in $9.10 \%$ (283/3110) samples. It has been reported in [36] that MYC-encoded protein is a multifunctional nucleolar phosphate protein, which acts as a transcription factor 
regulating target genes during the cell life cycle and cell transformation process.

Overexpression, mutation, translocation and rearrangement of MYC are closely related to the occurrence and development of a variety of cancers. It can be seen from Figure 3I that FBXW7 and MYC have a large edge weight value, so these two genes may likely have biological relevance. It has been reported in [37]that FBXW7 loss of function contributes to worse overall survival and is associated with accumulation of MYC in muscle invasive bladder cancer.

\section{Discussion}

This method identifies more new candidate gene sets for biological verification, and the findings indicate that the identified candidate gene sets have high biological relevance and statistical significance. The enrichment effect of the driver modules detected by ECSWalk is better than that by HotNet2 and MEXCOWalk algorithms among the nine types of cancer, especially in GBM, Melanoma, UCEC, NSCLC, and CML. The EGFR module, PPFIA1 module and TP53 module detected by ECSWalk have higher coverage than those by the other two algorithms. In particular, the PPFIA1 module detected by ECSWalk contains more PTPRS genes compared with the other two algorithms, which makes the PPFIA1 module more biologically and statistically significant. Besides, we found that the EGFR module has extremely high enrichment in UCEC(p-value: 1.09E-13), the TP53 module has extremely high enrichment in $\mathrm{CML}$ (p-value: 1.1E-11). The results are of theoretical guidance and practical value for cancer diagnosis, treatment and drug targets. There are certain limitations in this study, although the greedy strategy can accurately identify the 
driver genes belonging to the same module. This also leads to a decrease in the operating efficiency of the algorithm to a certain extent.

\section{Conclusion}

This study proposes a carcinogenic driver module detection algorithm (ECSWalk)

by integrating somatic mutation data and protein interaction network. This study firstly calculates the similarity between connected nodes in the protein interaction network. Then, a weighted network is created by calculating mutual exclusivity, coverage and topological structure similarity between mutation genes, and a restart random walk clustering method is utilized to detect the carcinogenic driver modules. Finally, an induced subgraph method is utilized to split the large modules, and a greedy strategy is utilized to expand the small modules to generate a set of driver modules. The experimental results show that the ECSWalk can detect more accurate driver modules than the other two algorithms. The driver modules detected by ECSWalk have a lower p-value by the DAVID and Go enrichment analysis, which indicates that the results of the algorithm have higher biological relevance and statistical significance. It also shows that ECSWalk can achieve good results by combining biological characteristics and complex network characteristics in detecting carcinogenic driver modules. Therefore, the application of complex network topology to biological networks is conducive to the study of the pathogenesis of cancer based on the inherent properties of the data itself, and it is also conducive to researchers and medical practitioners when carrying out research from the aspect of complex network topology. Besides, ECSWalk can identify the target gene set in some common cancers accurately, and analyze the biological relevance and statisti- 
cal significance of the driver modules, which thus helps to enrich our understanding

of the pathogenesis of cancer.

\section{Acknowledgements}

We thank Jihua Dong for her careful proofreading, and also thank Bing Zhou, Zhaoheng A, Mengdi Liu, Pengyu

Zhang and Haoru Zhou for their helpful advice and discussions.

\section{Funding}

The work was supported by the National Natural Science Foundation of China (Grant No.61972322), the Natural

Science Foundation of Shaanxi Province (Grant No. 2021JM-110) and the Humanities and Social Science Fund of

Ministry of Education of China (Grant No.18YJCZH190). The funders had no role in study design, data collection

and analysis, decision to publish, or preparation of the manuscript.

\section{Abbreviations}

ECSWalk: A carcinogenic driver module detection method based on a network model.

ICGC: International Cancer Genome Consortium

TCGA: The Cancer Genome Atlas.

HotNet2: An algorithm for finding significantly altered subnetworks in a large gene interaction network.

MEXCOWalk: Mutual exclusion and coverage based random walk to identify cancer modules.

PPI: Protein-protein interaction networks.

HINT+HI2012: A combination of high-quality protein-protein interactions from HINT and the recent HI-2012

set of protein-protein interactions.

SCC: Strongly connected component of the directed graph.

KL: Kullback-Leibler, a method of describing the difference between two probability distributions.

JS: Jensen-Shannon, an improved method based on $\mathrm{KL}$ divergence.

DAVID: The Database for Annotation, Visualization and Integrated Discovery.

TP: True Positive

FP: False Positive

TN: True Negative

FN: False Negative

\section{Availability of data and materials}

The data and materials we used can be download from https://github.com/raphael-group/hotnet2.(M.D.M.

Leiserson*, F. Vandin*, H.T. Wu, et al. (2014) Pan-Cancer Network Analysis Identifies Combinations of Rare

Somatic Mutations across Pathways and Protein Complexes. Nature Genetics 47, 106-114 (2015))

Ethics approval and consent to participate

Not applicable 


\section{Competing interests}

The authors declare that they have no competing interests.

\section{Consent for publication}

Not applicable

\section{Authors' contributions}

Conceive and design the experiments: HW ZC. Perform the experiments: HW ZC. Analyze the data: ZC YW.

Contribute reagents/materials/analysis tools: ZC YW QL. Write the paper: HW ZC. Consult on the final version of the paper and edit the paper: HW ZC HZ. The authors read and approve the final version of the manuscript.

\section{Authors' information}

Hao Wu, born in 1979, Ph.D., associate professor. His main research interests are within data mining, deep learning, computational bioinformatics, complex networks and complex diseases, particularly in cancer genomics and network biology. Email address: haowu@sdu.edu.cn.

Zhong-Li Chen, born in 1994, M.S. candidate. His main research interests are within computational bioinformatics and biological big data mining. Email address: czl@nwafu.edu.cn.

Ying-Fu Wu, born in 1998, M.S. candidate. His main research interests are within computational bioinformatics and biological big data mining. Email address: wuyingfunwsuaf@163.com.

Hong-Ming Zhang, born in 1979, Ph.D., professor. His main research interests are within spatial big data analysis and precision agriculture. Email address: zhm@nwsuaf.edu.cn.

Quan-Zhong Liu, born in 1978, Ph.D., associate professor. His main research interests are within bioinformatics and data mining. Email address: liuqzhong@nwsuaf.edu.cn.

\section{Author details}

${ }^{1}$ College of Information Engineering, Northwest A\&F University, Yangling, 712100, China. ${ }^{2}$ School of Software, Shandong University, Jinan, 250100, China. ${ }^{3}$ Tibet Center for Disease Control and Prevention, Lhasa, 850000 , China.

\section{References}

1. Mckeage, M., Shepherd, P., Yozu, M., D, R.L.: Tumour mutation profiling with high-throughput multiplexed genotyping: A review of its use for guiding targeted cancer therapy. Current Cancer Therapy Reviews 9(4), 236-244 (2013)

2. Yu, X., Zeng, T., Li, G.: Integrative enrichment analysis: a new computational method to detect dysregulated pathways in heterogeneous samples. BMC Genomics 16(1), 918 (2015)

3. Zhang, J., Wu, L., Zhang, S., Zhang, S.: Discovery of co-occurring driver pathways in cancer. BMC Bioinformatics 15(1), 1-14 (2014)

4. Zhao, J., Zhang, S., Wu, L., Zhang, X.: Efficient methods for identifying mutated driver pathways in cancer. Bioinformatics 28(22), 2940 (2012) 
5. Greenman, C., Stephens, P., Smith, R., Dalgliesh, G., Hunter, C., Bignell, H. Gand Davies, Teague, J., Butler, A., Stevens, C., Edkins, S., O'Meara, S., Vastrik, I., Schmidt, E., Avis, T., Barthorpe, S., Bhamra, G., Buck, G., Choudhury, B., Clements, J., Cole, J., Dicks, E., Forbes, S., Gray, K., Halliday, K., Harrison, R., Hills, K., Hinton, J., Jenkinson, A., Jones, D., Menzies, A., Mironenko, T., Perry, J., Raine, K.: Patterns of somatic mutation in human cancer genomes. Nature 446(7132), 153-158 (2007)

6. Vandin, F., Upfal, E., Raphael, B.: De novo discovery of mutated driver pathways in cancer. Genome Research 22(2), 175-181 (2012)

7. Leiserson, M., Blokh, D., Sharan, R., J. Raphael, B.: Simultaneous identification of multiple driver pathways in cancer. PLOS Computational Biology 9(5), 1003054 (2013)

8. Hou, J., Ma, J.: Dawnrank: discovering personalized driver genes in cancer. Genome Medicine 6(7), 5 (2014)

9. Srihari, S., Ragan, M.: Systematic tracking of dysregulated modules identifies novel genes in cancer. Bioinformatics 29(12), 1553-1561 (2013)

10. Wu, H., Gao, L., Dong, J., Yang, X.: Detecting overlapping protein complexes by rough-fuzzy clustering in protein-protein interaction networks. Plos One 9(3), 91856 (2014)

11. Miller, C., Settle, S., Sulman, E., Aldape, K., Milosavljevic, A.: Discovering functional modules by identifying recurrent and mutually exclusive mutational patterns in tumors. BMC Medical Genomics 4(1), 34 (2011)

12. $\mathrm{Wu}, \mathrm{H} .:$ Algorithm for detecting driver pathways in cancer based on mutated gene networks. Chinese Journal of Computers 41(6), 1400-1414 (2018)

13. Kim, Y., Cho, D., Dao, P., Przytycka, T.: Memcover: integrated analysis of mutual exclusivity and functional network reveals dysregulated pathways across multiple cancer types. Bioinformatics 31(12), 284-292 (2015)

14. Leiserson, M., Vandin, F., Wu, H., Dobson, J., Eldridge, J., Thomas, J., Papoutsaki, A., Kim, Y., Niu, B., McLellan, M., Lawrence, M., Gonzalez-Perez, A., Tamborero, D., Cheng, Y., Ryslik, G., Lopez-Bigas, N., Getz, G., Ding, L., Raphael, B.: Pan-cancer network analysis identifies combinations of rare somatic mutations across pathways and protein complexes. Nature Genetics 47(2), 106-114 (2015)

15. Reyna, M., Leiserson, M., Raphael, B.: Hierarchical hotnet: identifying hierarchies of altered subnetworks. Bioinformatics 34(17), 972-980 (2018)

16. Rafsan, A., Ilyes, B., Cesim, E., Evis, H., Hilal, K.: Mexcowalk: Mutual exclusion and coverage based random walk to identify cancer modules. Bioinformatics 36(3), 872-879 (2019)

17. Wu, H., Gao, L., Li, F., Yang, X., Kasabov, N.: Identifying overlapping mutated driver pathways by constructing gene networks in cancer. BMC Bioinformatics 16(5), 3 (2015)

18. Nepusz, T., Yu, H., Paccanaro, A.: Detecting overlapping protein complexes in protein-protein interaction networks. Nature Methods 9(5), 471-472 (2012)

19. Guo, M., Wang, S., Liu, X., Tian, Z.: Algorithm for predicting the associations between mirnas and diseases. Journal of Software 28(11), 3094-3102 (2017)

20. Tang, D., Zhu, Q., Yang, F., Chen, K.: Efficient cluster analysis method for protein sequences. Journal of Software 22(8), 1827-1837 (2011) 
21. Hou, Y., Duan, L., Li, L., Lu, L., Tang, C.: Search of genes with similar phenotype based on disease information network. Journal of Software 29(3), 721-733 (2018)

22. Zhang, Q., Li, M., Deng, Y.: A new structure entropy of complex networks based on tsallis nonextensive statistical mechanics. International Journal of Modern Physics C 27(10), 440-450 (2016)

23. Hofree, M., Shen, J., Carte, H., Gross, A., Ideker, T.: Network-based stratification of tumor mutations. Nature Methods 10, 1108-1115 (2013)

24. Li, F., Gao, L., Wang, B.: Detection of driver modules with rarely mutated genes in cancers. IEEE/ACM Transactions on Computational Biology and Bioinformatics 17(2), 390-401 (2020)

25. Wang, B., Wang, M., Li, X., M, Y., Liu, L.: Variations in the wnt/ $\beta$-catenin pathway key genes as predictors of cervical cancer susceptibility. Pharmacogenomics and personalized medicine 13, 157-165 (2020)

26. Katherine, S., Noriko, U., Wiljan, H., Michel, L., Bouchard, M.: Inactivation of lar family phosphatase genes ptprs and ptprf causes craniofacial malformations resembling pierre-robin sequence. Development 140(16), 3413-3422 (2013)

27. Mana, G., Clapero, F., Panieri, E., Panero, V., Böttcher, R., Tseng, H., Saltarin, F., Astanina, E., Wolanska, K., Morgan, M., Humphries, M., Santoro, M., Serini, G., Valdembri, D.: Ppfia 1 drives active $\alpha 5 \beta 1$ integrin recycling and controls fibronectin fibrillogenesis and vascular morphogenesis. Nature Communications 7(1), $13546(2016)$

28. Li, H., Liu, L., Liu, C., Zhuang, J., Zhou, C., Yang, J., Gao, C., Liu, G., Lv, Q., Sun, C.: Deciphering key pharmacological pathways of qingdai acting on chronic myeloid leukemia using a network pharmacology-based strategy. Med Sci Monit 24, 5668-5688 (2018)

29. Xia, Y., Zeng, Y., Zhang, M., Liu, P., Liu, F., Zhang, H., He, C., Sun, Y., Zhang, J., Zhang, C., Song, L., Ding, C., Tang, Y., Yang, Z., Yang, C., Wang, P., Guan, K., Xiong, Y., Ye, D.: Tumor-derived neomorphic mutations in asxl1 impairs the bap1-asxl1-foxk1/k2 transcription network. Protein \& Cell (2020)

30. Wang, X., Xi, X., Wu, J., Wan, Y., Hui, H., Cao, X.: Microrna-206 attenuates tumor proliferation and migration involvingthe downregulation of notch3 in colorectal cance. Oncology Reports 33(3), 1402-1410 (2015)

31. Catarina, R., Susana, R., Claudia, G., Domingos, H.: Two notch ligands, dll1 and jag1, are differently restricted in their range of action to control neurogenesis in the mammalian spinal cord. Plos One 5(11), 15515 (2010)

32. Amrich, C., Davis, C., Rogal, W., Shirra, M., Heroux, A., Gardner, R., Arndt, K., VanDemark, A.: Cdc73 subunit of paf1 complex contains c-terminal ras-like domain that promotes association of paf1 complex with chromatin. The Journal of biological chemistry 287(14), 10863-75 (2012)

33. Mueller, C., Jaehning, J.: Ctr9, rtf1, and leo1 are components of the paf1/rna polymerase ii complex. Molecular and Cellular Biology 22(7), 1971-1980 (2002)

34. Tsujino, I., Nakanishi, Y., Shimizu, T., Obana, Y., Ohni, S., Takahashi, N., Nemoto, N., Hashimoto, S.: 999 correlation between differences in the increase in mapk (erk1/2) activity due to driver mutations and prognosis in non-small-cell lung cancer. European Journal of Cancer 48, 241-241 (20012)

35. Schwickart, M., Huang, X., Lill, J., Liu, J., Ferrando, R., French, D., Maecker, H., O’Rourke, K., Bazan, F., 
Eastham-Anderson, J., Yue, P., Dornan, D., Huang, D., Dixit, V.: Deubiquitinase usp9x stabilizes mcl1 and promotes tumour cell survival. Nature 463(7277), 103-107 (2010)

36. Sabò, A., Kress, T., Pelizzola, M., De, P., Gorski, M., Tesi, A., Morelli, M., Bora, P., Doni, M., Verrecchia, A., Tonelli, C., Fagà, G., Bianchi, V., Ronchi, A., Low, D., Müller, H., Guccione, E., Campaner, S., Amati, B.:

Selective transcriptional regulation by myc in cellular growth control and lymphomagenesis. Nature $\mathbf{5 1 1}$,

488-492 (2014)

37. Matumoto, T., Chen, Y., Contreras-Sanz, A., Ikeda, K., Schulz, G., Gao, J., Oo, H., Roberts, M., Costa, J., Nykopp, T.: Fbxw7 loss of function contributes to worse overall survival and is associated with accumulation of myc in muscle invasive bladder cancer. Urologic Oncology: Seminars and Original Investigations 38(12), 904-905 (2010)

\section{Figures}

Figure 1: Comparison of enrichment effect

Figure 2: Comparison of modules accuracy A) ACC values, B) F-measure values

Figure 3: Driver modules detected by ECSWalk.

(The genes in the rough edge represent the names of the dysregulated modules.

The size of the circle is proportional to the mutation frequency, and the thickness of the line segment is proportional to the weight between nodes.)

Figure 4: Gene interaction diagram 
Table 1: EGFR module

\begin{tabular}{clccc}
\hline Algorithm & \multicolumn{1}{c}{ Gene set } & Number of modules & Coverage & P-Value \\
\hline \multirow{2}{*}{ HotNet2 } & EGFR ERBB2 AREG ELF3 & 7 & $17.94 \%$ & $6.9 \mathrm{E}-06$ \\
& ERBB4 LRIG1 OSMR & & & \\
& ERBB2 CDKN2A FLNA ERBB4 & & & \\
MEXCOWalk & ATM MCM2 IGF1R MDM4 & 16 & $45.98 \%$ & $8.1 \mathrm{E}-07$ \\
& PHF17 VHL NPM1 CDH1 & & & \\
& STK11 MDM2 EGFR TLN1 & & & \\
& PIK3CA ERBB2 EGFR TLN1 & & $47.85 \%$ & $1.09 \mathrm{E}-13$ \\
ECSWalk & $\begin{array}{l}\text { PIK3R1 CTNNB1 IGF1R NEDD9 } \\
\text { APC VARS2 HRAS NRAS }\end{array}$ & 12 & \\
\hline
\end{tabular}

Table 2: PPFIA1 module

\begin{tabular}{ccccc}
\hline Algorithm & Gene set & Number of modules & Coverage & P-Value \\
\hline HotNet2 & N/A & N/A & N/A & N/A \\
MEXCOWalk & PPFIA1 PPP2R1A PTPRF & 3 & $6.82 \%$ & N/A \\
ECSWalk & PTPRS PPFIA1 PPP2R1A PTPRF & 4 & $7.72 \%$ & N/A \\
\hline
\end{tabular}


Table 3: TP53 module

\begin{tabular}{|c|c|c|c|c|}
\hline Algorithm & Gene set & Number of modules & Coverage & P-Value \\
\hline HotNet2 & $\begin{array}{l}\text { CCND1 CDKN2A CUL9 NPM1 } \\
\text { PTEN TP53 AGBL2 ALS2CR8 } \\
\text { AMOTL1 ANKRD12 BRPF1 CACHD1 } \\
\text { CARNS1 CDKN2AIP CELSR3 CHD8 } \\
\text { EPHA3 HECW2 IFT140 IWS1 } \\
\text { MAGI2 MAST3 MDM4 MLL5 } \\
\text { PLEKHA8 PRDM2 PRKRIR SCAPER } \\
\text { SETD2 SMG1 SMG5 SMG7 } \\
\text { SNRK SPTBN2 STK11IP STRADA } \\
\text { SWAP70 TEP1 TRIP12 TTLL5 } \\
\text { UACA ZFP91 ZMIZ1 ZNF227 } \\
\text { ZNF668 }\end{array}$ & 45 & $68.39 \%$ & 3.84E-6 \\
\hline MEXCOWalk & $\begin{array}{l}\text { E4F1 PTGS2 BMP1 ELL } \\
\text { PPP1R13L WDR33 SMYD2 HINFP } \\
\text { HIPK2 EGR1 KAT8 STK4 } \\
\text { NOC2L EHMT1 CUL9 SNRPN } \\
\text { CABLES1 WT1 WWOX CCT5 } \\
\text { ARID3A HSPA9 ZNF384 RFWD2 } \\
\text { TOP1 PLK3 RNF20 ERCC6 } \\
\text { TOPORS TP53 RB1CC1 CDKN1B } \\
\text { CUL1 KDM5A BRCA1 CTNNB1 } \\
\text { CDK4 CCND1 RB1 CDK6 }\end{array}$ & 40 & $49.58 \%$ & $5.6 \mathrm{E}-8$ \\
\hline ECSWalk & $\begin{array}{l}\text { BLM CSNK2A1 PTK2 WDR33 } \\
\text { ING1 USP7 ING5 BRCA1 } \\
\text { STAT1 UBE3A EP300 MDM2 } \\
\text { MDM4 MED1 ATM CHD3 } \\
\text { CREBBP WRN CUL9 IKBKB } \\
\text { WT1 HSP90AA1 CHEK2 BCL2L1 } \\
\text { CDK4 CDK6 ZNF384 CDKN2A } \\
\text { XPO1 SIN3A HDAC2 HTT } \\
\text { DDX5 TP53 AKT1 NPM1 } \\
\text { RB1 TP53BP1 PTEN SMARCA4 }\end{array}$ & 40 & $70.09 \%$ & $3.25 \mathrm{E}-20$ \\
\hline
\end{tabular}


Figures

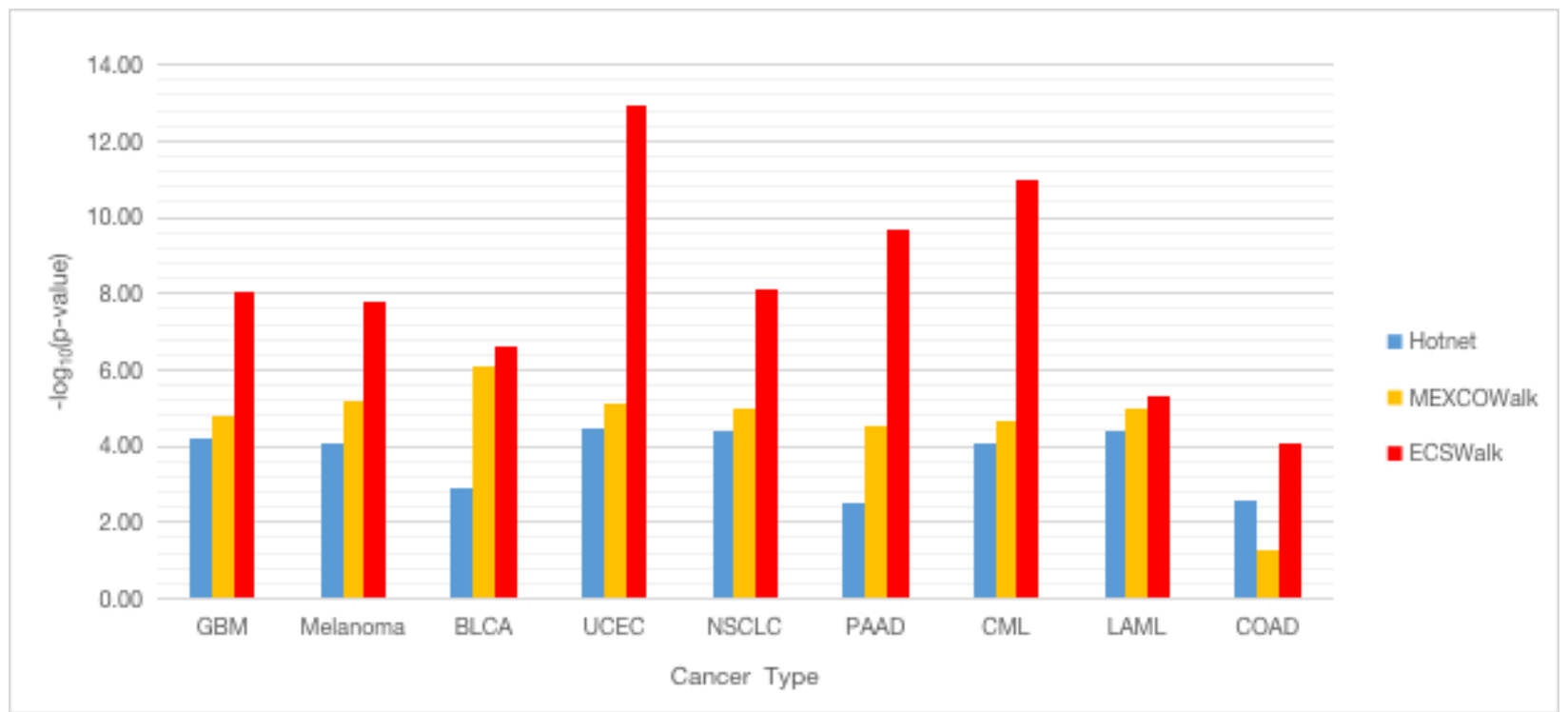

Figure 1

Comparison of enrichment effect 

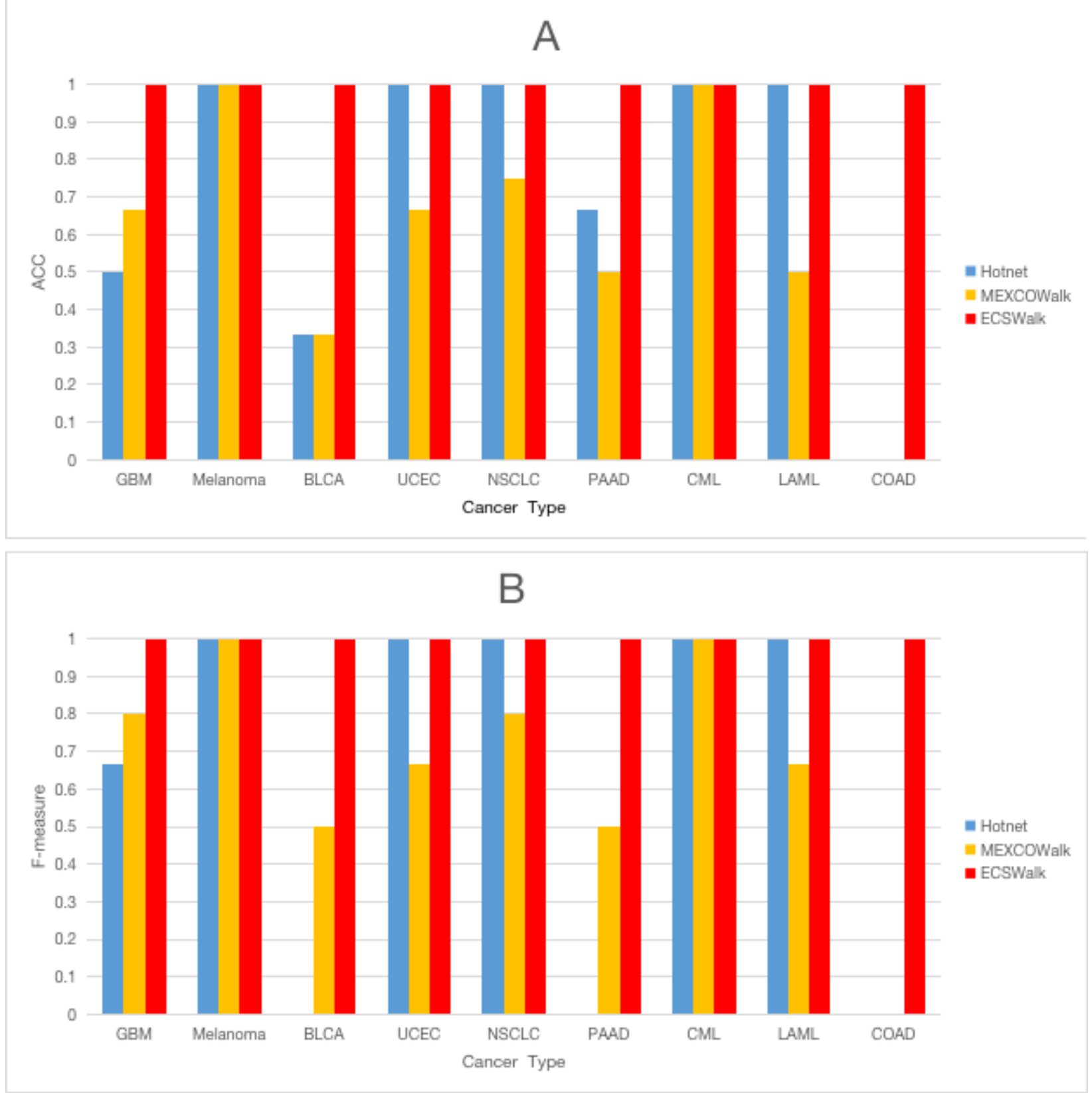

Figure 2

Comparison of modules accuracy A) ACC values, B) F-measure values 


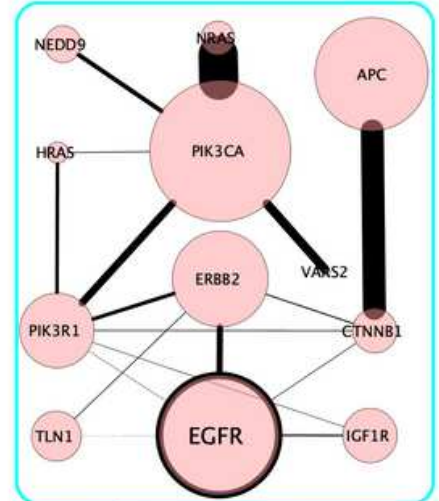

A) EGFR module

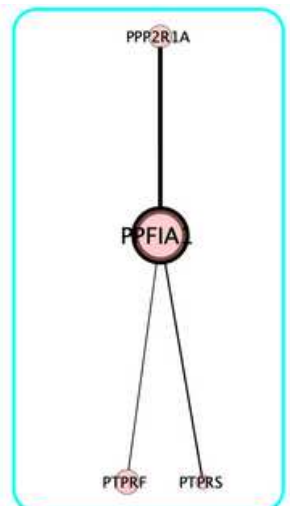

B) PPFIAI module

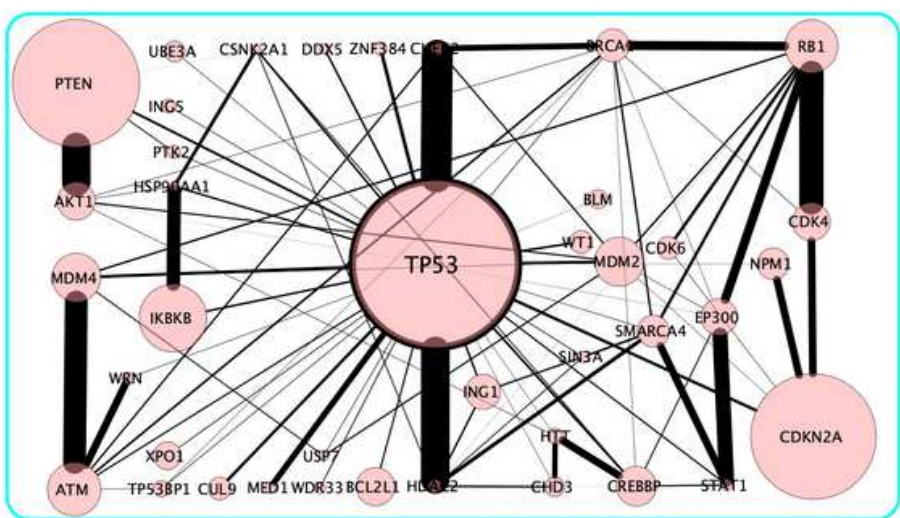

C) TP53 module

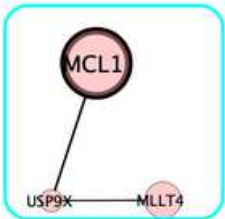

G) MAP3K1 module H) MCL1 module

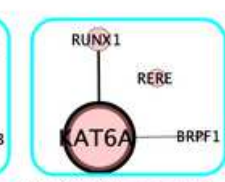

N) KAT6A module

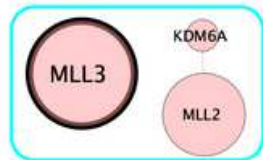

O) MLL3 module

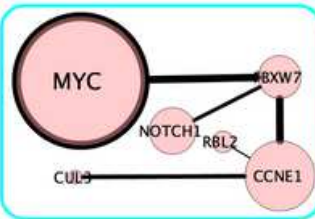

I) MYC module

P) VHL module

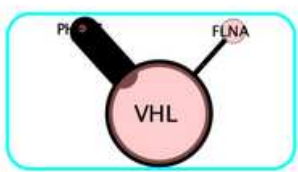

\section{Figure 3}

Driver modules detected by ECSWalk. (The genes in the rough edge represent the names of the dysregulated modules. The size of the circle is proportional to the mutation frequency, and the thickness of the line segment is proportional to the weight between nodes.) 


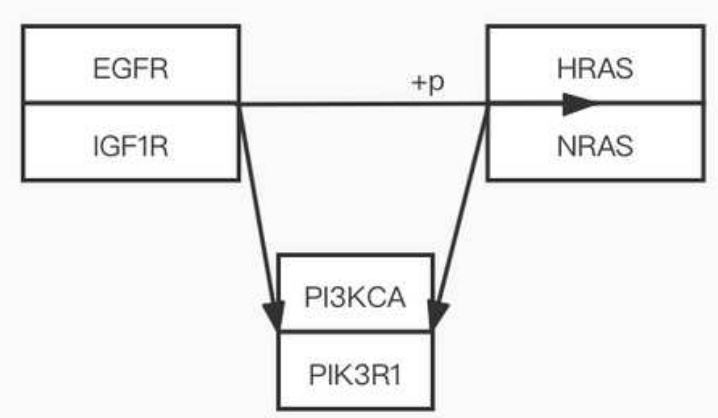

a) ErbB signal pathway

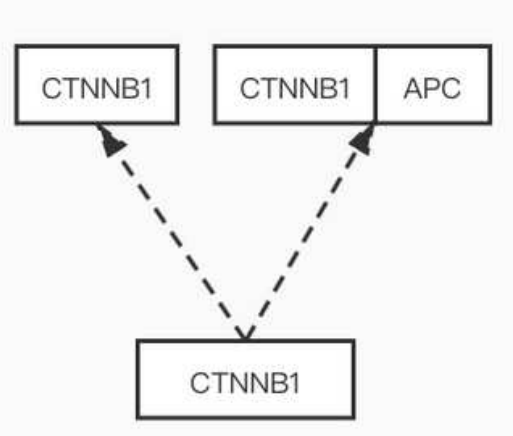

b) Wnt signal pathway

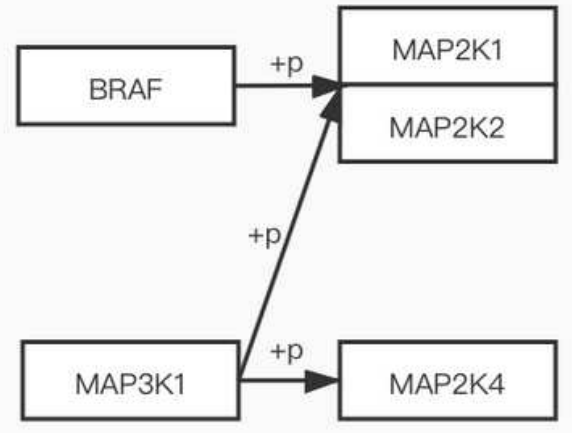

c) MAPK signal pathway

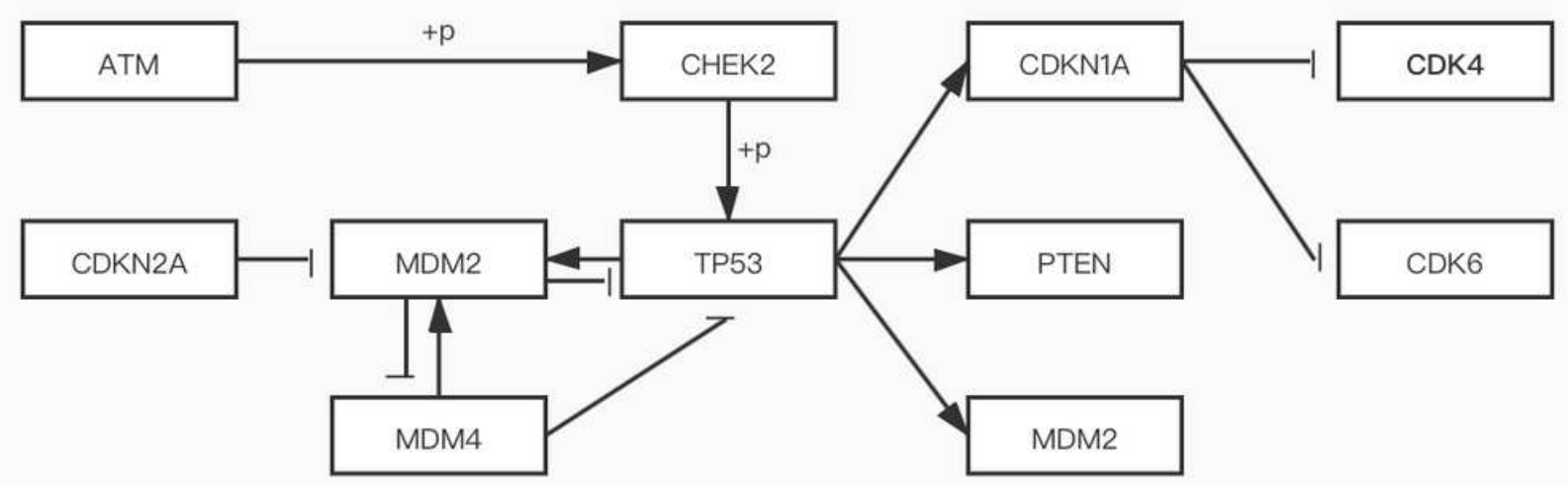

d) P53 signal pathway

Figure 4

Gene interaction diagram 